\title{
Fachbericht «Orale oder parenterale Behandlung des Eisenmangels»
}

Swiss Medical Board

Korrespondenz: Susanna Marti Calmell Sekretariat Trägerschaft Swiss Medical Board Stampfenbachstrasse 30 Postfach

CH-8090 Zürich

Tel. 0432595211

info[at]medical-board.ch
Das Swiss Medical Board ist der Frage einer adäquaten Behandlung bei schwerem Eisenmangel nachgegangen. In seinem neuesten Bericht empfiehlt das Expertengremium des Swiss Medical Board die Anwendung intravenöser Eisenpräparate nach sorgfältiger Abklärung und bei gleichzeitiger Behandlung der Ursachen des Eisenmangels. Die Trägerschaft hat den Bericht zur Kenntnis genommen und unterbreitet ihn einer Post-Publication Review.

Eisen ist ein bedeutendes Element für den menschlichen Körper, da es zentraler Baustein des roten Blutfarbstoffs Hämoglobin und damit für die Versorgung der Zellen mit Sauerstoff verantwortlich ist. Darüber hinaus spielt Eisen in zahlreichen Stoffwechselprozessen eine wichtige Rolle. Verstärkter Eisenmangel führt zu einer erheblichen Beeinträchtigung der körperlichen und geistigen Leistungsfähigkeit (mit Müdigkeit, Kopfschmerzen und Konzentrationsstörungen) und kann zu einer Anämie (Blutarmut) führen. Zudem kann Eisenmangel insbesondere bei chirurgischen Interventionen einen erheblichen Risikofaktor darstellen.

Obwohl durch eine eisenreiche Ernährung oder Massnahmen einer verbesserten Eisenresorption einer Unterversorgung in der Regel vorgebeugt werden kann, muss bei schwerem Mangel Eisen medikamentös substituiert werden. Das SMB hat untersucht, ob in solchen Fällen die Verabreichung von Eisenpräparaten mittels Infusion gegenüber einer oralen Therapie für den Patienten hinsichtlich der Wirksamkeit und der Lebensqualität Vorteile bringt. Dabei wurden auch mögliche unerwünschte Wirkungen und das Kosten-Wirksamkeits-Verhältnis der Behandlung betrachtet.

Aufgrund der vorliegenden Literatur kommt das Expertengremium zum Schluss, dass beide Verabreichungsformen die Symptome des Eisenmangels positiv beeinflussen können. Im Falle eines schweren Eisenmangels wird festgestellt, dass die intravenöse Therapie die positiven Auswirkungen schneller und quantitativ ausgeprägter erreicht. Das Expertengremium empfiehlt daher:

- Bei Patienten mit einem symptomatischen, schweren Eisenmangel oder einer Eisenmangelanämie ist nach sorgfältiger Abklärung eine parenterale Substitutionstherapie sinnvoll. Bei der Durchführung sind eine entsprechende Überwachung und Interventionsbereitschaft in Hinsicht auf eventuelle Nebenwirkungen sicherzustellen.

- Gleichzeitig ist eine Behandlung der Ursache(n) des Eisenmangels einzuleiten.

- Darüber hinaus sollte immer geprüft werden, ob Patienten im Zusammenhang mit einer chirurgischen Intervention von einer parenteralen Eisensubstitutionstherapie profitieren können.

Weitere Informationen zum Swiss Medical Board generell: www.swissmedicalboard.ch; zu den Berichten des Fachgremiums Swiss Medical Board: $\rightarrow$ Fachbericht $\rightarrow$ Abgeschlossene Berichte.

\section{Post-Publication Review zum Fachbericht «Orale oder parenterale Behandlung des Eisenmangels»}

Der Fachbericht gibt die auf aktueller medizinischer Evidenz basierende Einschätzung des unabhängigen Expertengremiums Swiss Medical Board wieder. Die Trägerschaft des Swiss Medical Board hat den Bericht zur Kenntnis genommen und möchte interessierte Kreise zu einer Fachdiskussion im Sinne einer Post-Publication Review einladen.

Ihren Beitrag nehmen wir gerne entgegen unter: info[at]medical-board.ch 